\title{
Core Needle Biopsy Specimens Are More Appropriate than Surgical Specimens for Evaluating the Expression of Phosphoproteins as Biological Markers in Invasive Breast Cancer
}

\author{
HYUN-AH KIM ${ }^{1 *}$, JI HYUN KIM ${ }^{1 *}$, HYANG SUK CHOI ${ }^{1}$, YUN GYOUNG KIM ${ }^{1}$, SEOK JOON LEE ${ }^{1}$, \\ KANG HEE HAN ${ }^{2}$, MIN KI SEONG1, HYE SIL SEOL ${ }^{2}$ and WOO CHUL NOH ${ }^{1}$ \\ Departments of ${ }^{1}$ Surgery, and ${ }^{2}$ Pathology, Korea Cancer Center Hospital, \\ Korea Institute of Radiological and Medical Sciences, Seoul, Republic of Korea
}

\begin{abstract}
Background/Aim: To evaluate whether core needle biopsy specimens (CNBS) would be better than surgical specimens (SS) for evaluating phosphoproteins as biological markers in breast cancer. Materials and Methods: This retrospective analysis included a total of 99 patients who had invasive breast cancer and were treated surgically between January 2012 and July 2013. The expression of phosphorylated ribosomal protein S6-kinase-1 (p-S6K1), phosphorylated protein kinase $B(p-A K T)$, and estrogen receptor (ER) were assessed immunohistochemically on both $C N B S$ and SS for each patient. Results: The expression rate of phosphoprotein at any intensity was higher on CNBS than on $S S$ ( $p$-S6K1, 99.0\% versus $59.6 \%$; $p$-AKT, $94.9 \%$ versus $56.6 \%)$. $p$-S6Kland p-AKT expression was classified as negativity (absence to weak staining) or positivity (moderate to strong staining) for further analysis. For p-S6K1, 24.2\% of patients showed positivity on CNBS and negativity on SS, while $7 \%$ of the patients showed negativity on CNBS and positivity on SS. For p-AKT, 70.7\% of the patients showed positivity on CNBS and negativity on SS, while only $1.0 \%$ of patients showed negativity on CNBS and positivity on SS. Conclusion: CNBS may be the more appropriate specimen type for immunohistochemical examination of phosphoprotein expression in invasive breast cancer.
\end{abstract}

\footnotetext{
*These Authors contributed equally to this study.

Correspondence to: Woo Chul Noh, MD, Ph.D., Department of Surgery, Korea Cancer Center Hospital, Korea Institute of Radiological and Medical Sciences, Seoul, Republic of Korea, 2154 Gongneung-dong, Nowon-ku, Seoul 139-706, Korea. Tel: +82 29701221, Fax: +82 29701221, e-mail: nohwoo@kcch.re.kr
}

Key Words: Phosphoprotein expression, invasive breast cancer, core needle biopsy specimens.
Protein phosphorylation is an important step for modulating the activities of many signaling molecules that play key roles in cell proliferation (1). To assess the activities of signaling pathways and the effects of cell signal targeting agents, it is important to measure the expression of phosphorylated proteins using an accurate and feasible method. In breast cancer, the phosphoinositide 3-kinase/protein kinase $\mathrm{B} /$ mechanistic target of rapamycin (PI3K/AKT/mTOR) signaling pathway is one of the major cell signaling pathways that has been investigated in order to develop targeted agents. The activation of this pathway is related to poor prognosis and treatment. Inhibitory agents that target each step of the PI3K/AKT/mTOR pathway are under active development as treatment for breast cancer (2).

Ribosomal protein S6-kinase-1 (S6K1) is a downstream regulator of the $\mathrm{PI} 3 \mathrm{~K} / \mathrm{AKT} / \mathrm{mTOR}$ signaling pathway. S6K1 is a core regulator of cell survival and proliferation, metabolism, migration, and angiogenesis (3-5). In breast cancer, S6K1 has been investigated as a prognostic and predictive marker of mTOR-targeted therapy (6). Phosphorylated-S6K1 (p-S6K1) is an active form of $\mathrm{S} 6 \mathrm{~K} 1$ and is related to poor prognosis and resistance to endocrine therapy $(7,8)$. Both phosphorylatedAKT (p-AKT) and p-S6K1 are surrogating markers for detecting activation of the $\mathrm{PI} 3 \mathrm{~K} / \mathrm{AKT} / \mathrm{mTOR}$ pathway. However, questions have been raised regarding the accuracy and reproducibility of immunohistochemical evaluation of $\mathrm{p}$ S6K1 and p-AKT expression.

One possible reason that immunohistochemistry results are inconsistent for p-S6K1 and p-AKT is the variability of the interval from the time that the tissue is obtained to the time of fixation in surgery (9). Immunohistochemical evaluation of phosphorylated protein expression on paraffin blocks is critically influenced by the time interval from tissue acquisition to fixation $(10,11)$. In a pre-clinical investigation that used colon cancer xenografts, p-AKT did not stain in tissue after samples were allowed to stand for $30 \mathrm{~min}$ at 
room temperature, even though it has been present in the fresh sample. The half-life of p-AKT was $20 \mathrm{~min}$, while the half-life of AKT was $180 \mathrm{~min}$ (11).

Theoretically, shorter time intervals from tissue acquisition to fixation could guarantee better-quality results in immunohistochemical measurements of phosphoprotein. In most studies, phosphorylated protein was measured in surgical specimens (SSs), and the time interval from surgical removal of the tumor to fixation is variable in actual practice in the surgical theatre. In contrast, core needle biopsy specimens (CNBSs) are fixed immediately after biopsy in usual clinical practice. The substantively different fixation periods of CNBSs and SSs may lead to differences in pS6K1 and p-AKT measurements. Therefore, we sought to compare the expression of $\mathrm{p}-\mathrm{S} 6 \mathrm{~K} 1$ and $\mathrm{p}-\mathrm{AKT}$ in paraffinembedded CNBS and SS samples from the same patients.

\section{Patients and Methods}

Study population. Patients who underwent a surgical procedure between January 2012 and July 2013 were identified in the Korea Cancer Center Hospital Breast Cancer Center (KCCHBCC) database. The inclusion criteria were patients who had primary invasive ductal or lobular breast cancer, as well as eligible paraffin blocks for both a CNBS and an SS. Patients with breast cancer that was metastatic at the time of the initial diagnosis and those who received neo-adjuvant chemotherapy were excluded from this analysis. Patients with T3-T4 disease and distant metastasis were also excluded to avoid biases caused by central necrosis of a large mass. A total of 99 patients with invasive breast cancer were included in this retrospective study. Histological type, histological grade, TNM stage, hormonal receptor status, human epidermal growth factor receptor 2 (HER2) status, and other clinicopathological characteristics were collected from the KCCHBCC database and an electric medical records review. Human epidermal growth factor receptor 2 (HER2) positivity was defined as $3(+)$ on immunohistochemical staining or HER2 gene amplification by fluorescence in situ hybridization or silver in situ hybridization. The study protocol was approved by the Institutional Review Board of Korea Institute of Radiological and Medical Sciences (K-1601-002-007).

Immunohistochemistry and molecular subtype. Formalin-fixed, paraffin-embedded tumor tissue blocks were used for immunohistochemistry. The tissue sections of one CNBS and one SS from each patient were immunohistochemically stained with appropriate antibodies for $\mathrm{p}-\mathrm{S} 6 \mathrm{~K} 1, \mathrm{p}-\mathrm{AKT}$, and estrogen receptor (ER).

The p-S6K1 expression status was assessed by immunohistochemistry with a mouse monoclonal antibody against p-S6K1 (dilution 1:500; Cell Signaling Technology, Inc., Danvers, MA, USA). The p-AKT expression was immunohistochemically stained using a mouse monoclonal antibody against p-AKT (dilution 1:50; Thermo Scientific, Waltham, MA, USA). The intensity score was as follows: +1 , weak staining; +2 , intermediate staining; and +3 , strong staining. The absence or weak expression of p-S6K1 or p$\mathrm{AKT}$ was classified as negativity, and moderate or strong expression was classified as positivity.
ER positivity was defined as positive staining with antibody (dilution 1:200; Thermo Scientific) of $\geq 10 \%$ of nuclei in 10 highpower fields.

Statistical analysis. Fisher's exact test was used to compare variables between groups. $p$-Values of less than 0.05 were considered statistically significant.

\section{Results}

A total of 99 patients were investigated, of whom one had bilateral breast cancer. The mean age was $52.4 \pm 10.1$ years. Most patients $(94.9 \%)$ had invasive ductal carcinoma. The mean tumor size was $1.7 \pm 0.8 \mathrm{~cm}$. Regarding TNM stage, most patients had stage I or II disease (91.9\%). The clinicopathological characteristics of the study population are shown in Table I.

For $\mathrm{p}-\mathrm{S} 6 \mathrm{~K} 1$, the expression rate at any intensity was $99.0 \%$ on CNBS and $59.6 \%$ on SS. For p-AKT, the expression rate at any intensity was $94.9 \%$ on CNBSs and $56.6 \%$ on SSs. For ER, the expression rate at any intensity was $77.8 \%$ on CNBS and $76.8 \%$ on SS. After applying the cut-off values described in the Materials and Methods, the positivity rate for p-S6K1 was $32.3 \%(32 / 99)$ by CNBS and $15.1 \%(15 / 99)$ by SS, for p-AKT they were $76.8 \%(76 / 99)$ and only $7 \%$ (7/99), respectively. In contrast with the highly different rates of $\mathrm{p}-\mathrm{S} 6 \mathrm{~K} 1$ and $\mathrm{p}-\mathrm{AKT}$ expression that were observed for CNBS and SS samples, ER was expressed in $77.8 \%$ and $76.8 \%$ of CNBSs and SSs, respectively (Table II).

Regarding p-S6K1, 24.2\% (24/99) of patients showed positivity on the CNBS and negativity on the SS, while $7 \%$ (7/99) of patients showed negativity on the CNBS and positivity on the SS. Regarding p-AKT, 70.7\% (70/99) of patients showed positivity on the CNBS and negativity on the SS, while only $1.0 \%$ (1/99) of patients showed negativity on the CNBS and positivity on the SS. Unlike the p-S6K1 and p-AKT findings, ER expression results were well matched for CNBSs and the corresponding SSs $(p<0.001$, Table II).

\section{Discussion}

In this study, we found that $\mathrm{p}-\mathrm{S} 6 \mathrm{~K} 1$ and $\mathrm{p}-\mathrm{AKT}$ were expressed at higher rates on CNBSs than on SSs from the same patients. In contrast, the expression of ER, which is not a phosphoprotein, was very similar in both CNBSs and SSs. To the best of our knowledge, this is the first report to have compared p-S6K1 and p-AKT expression between CNBSs and SSs from patients with invasive breast cancer.

The higher expression rates of $\mathrm{p}-\mathrm{S} 6 \mathrm{~K} 1$ and $\mathrm{p}-\mathrm{AKT}$ on CNBS samples than on SS samples is probably explained by the occurrence of dephosphorylation during the time interval between tissue sampling and fixation. Because only a few seconds are spent placing CNBSs into preservative solution, 
Table I. Clinicopathological features of the study population $(N=99)$.

\begin{tabular}{lc}
\hline Variable & No. of patients $(\%)$ \\
\hline Histological type & \\
Invasive ductal carcinoma & $94(94.9)$ \\
Invasive lobular carcinoma & $5(5.1)$ \\
Histological grade & \\
Grade 1 & $23(23.2)$ \\
Grade 2 & $44(44.4)$ \\
Grade 3 & $29(29.3)$ \\
Unknown & $3(3.3)$ \\
T-Stage & \\
T1 & $73(73.7)$ \\
T2 & $26(26.3)$ \\
N-Stage & \\
N0 & $68(68.7)$ \\
N1 & $23(23.2)$ \\
N2 & $7(7.1)$ \\
N3 & $1(1.0)$ \\
TNM stage & \\
Stage I & $56(56.6)$ \\
Stage II & $35(35.4)$ \\
Stage III & $8(8.9)$ \\
Operation & \\
Total mastectomy & $30(30.3)$ \\
Breast-conserving surgery & $69(69.7)$ \\
Subtype & \\
ER or PR-positive/HER2-negative & $61(61.6)$ \\
ER or PR-positive /HER2-positive & $15(15.2)$ \\
ER or PR-negative/HER2-positive & $9(10)$ \\
ER or PR-negative /HER2-negative & $14(14.1)$ \\
\hline
\end{tabular}

ER: Estogen receptor; PR: progestetone receptor; HER2: human epidermal growth factor receptor 2 .

there is little time for dephosphorylation to affect proteins in CNBSs. For SSs, however, at least a few minutes pass between vascular ligation and the placement of specimens into fixative solution. Our findings are consistent with a previous analysis, in which immunohistochemical staining for $\mathrm{p}$-Ser ${ }^{473}$-AKTwas only observed in tumor samples that had been obtained as biopsies, and no staining was observed in tumors that had been obtained as surgically resected samples (11). Regarding p-S6K1, a few investigators have reported that delayed fixation may lead to loss of phosphorylation $(12,13)$. Dephosphorylation may also explain the observation that the large majority of the discordant cases in our study exhibited positivity in the CNBS and negativity in the SS, instead of negativity in the CNBS and positivity in the SS. Indeed, 77.4\% (24/31) of discordant cases for p-S6K1 and 98.6\% (70/71) of discordant cases for p-AKT showed positivity in the CNBS and negativity in the SS. Taken together, our findings show that p-S6K1 and p-AKT in breast cancer samples could be dephosphorylated as time passes.
Table II. Expression of phosphorylated ribosomal protein S6-kinase-1 (p-S6K1), phosphorylated-protein kinase B ( $p$-AKT), and estrogen receptor $(E R)$ in surgical specimen and on core needle biopsy specimen

\begin{tabular}{lcccc}
\hline & \multicolumn{2}{c}{ Core needle biopsy specimen, $\mathrm{n}$} & \multirow{2}{*}{$p$-Value } \\
\cline { 2 - 3 } & Negative & Positive & Total & \\
\hline Surgical specimen, $\mathrm{n}$ & & & & \\
p-S6K1 & & & & \\
$\quad$ Negative & 60 & 24 & 84 & 0.059 \\
$\quad$ Positive & 7 & 8 & 15 & \\
$\quad$ Total & 67 & 32 & 99 & \\
p-AKT & & & & \\
Negative & 22 & 70 & 92 & 0.485 \\
Positive & 1 & 6 & 7 & \\
$\quad$ Total & 23 & 76 & 99 & \\
ER & & & & \\
$\quad$ Negative & 22 & 1 & 23 & $<0.001$ \\
Positive & 0 & 76 & 76 & \\
$\quad$ Total & 22 & 77 & 99 & \\
\hline
\end{tabular}

The strongest point of our analysis is the use of a paired CNBS and SS from each patient. As is well known, breast cancer is a very heterogeneous disease, that may lead to confusion in interpretation of study results. However, because the CNBS and SS were obtained from the same patients, biases introduced by the heterogeneous clinicopathological characteristics of breast cancer were overcome in the present study. The limitations to this study include our inability to analyze survival according to differences in the expression of p-S6K1 and p-AKT, as a result of the small number of events.

In the current era of targeted therapy, it is important to find new targets or prognostic markers in order to develop treatment strategies that are appropriate for patients with individual breast cancer. Both p-S6K1 and p-AKT have been investigated as promising markers in pre-clinical studies. However, they have not been recommended clinically because their results differed across studies. In the present study, we showed that CNBS could be a better choice than $\mathrm{SS}$ for examining the expression of $\mathrm{p}-\mathrm{S} 6 \mathrm{~K} 1$ or $\mathrm{p}-\mathrm{AKT}$ in patients with breast cancer. Considering that most breast cancer cases are actually diagnosed via CNB, using such samples could be a feasible, appropriate, and easily obtained choice for marker studies in many clinical trials.

\section{Acknowledgements}

This study was supported by a grant from the Korea Institute of Radiological and Medical Sciences (KIRAMS), funded by the Ministry of Science, ICT and Future Planning, Republic of KOREA (1711045543/50462-2017). 


\section{References}

1 Hunter T: A thousand and one protein kinases. Cell 50: 823-829, 1987.

2 Shou J, Massarweh S, Osborne CK, Wakeling AE, Ali S, Weiss $\mathrm{H}$ and Schiff R: Mechanisms of tamoxifen resistance: increased estrogen receptor-HER2/Neu cross-talk in ER/HER2-positive breast cancer. J Natl Cancer Inst 96(12): 926-935, 2004.

3 Fingar DC and Blenis J: Target of rapamycin (TOR): An integrator of nutrient and growth factor signals and coordinator of cell growth and cell-cycle progression. Oncogene 23: 31513171, 2004.

4 Shaw RJ and Cantley LC: Ras, PI (3)K and mTOR signaling controls tumour cell growth. Nature 441: 424-430, 2006.

5 Meric-Bernstam F and Gonzalez-Angulo AN: Targeting the mTOR signaling network for cancer therapy. J Clin Oncol 27: 2278-2287, 2009.

6 Noh WC, Mondesire WH, Peng J, Jian W, Zhang H, Dong J, Mills GB, Hung MC and Meric-Bernstam F: Determinants of rapamycin sensitivity in breast cancer cells. Clin Cancer Res 10(3): 1013-1023, 2004.

7 Noh WC, Kim YH, Kim MS, Koh JS, Kim HA, Moon, NM and Paik NS: Activation of the mTOR signaling pathway in breast cancer and its correlation with the clinicopatologic variables. Brest Cancer Research and Treatment 110(3): 477-483, 2008.

8 Kim EK, Kim HA, Koh JS, Kim MS, Kim KI, Lee JI, Moon NM, Ko E and Noh WC: Phosphorylated S6K1 is a possible marker for endocrine therapy resistance in hormone receptorpositive breast cancer. Breast Cancer Research and Treatment. 126(1): 93-99, 2011.
9 Bai Y, Tolles J, Cheng H, Siddiqui S, Gopinath A, Pectasides E, Camp RL, Rimm DL and Molinaro AM: Quantitative assessment shows loss of antigenic epitopes as a function of pre-analytic variables. Lab Invest 91: 1253-1261, 2011.

10 Start RD, Cross SS and Smith JHF: Assessment of specimen fixation in a surgical pathology service. J Clin Pathol 45: 546$547,1992$.

11 Baker AF, Dragovich T, Ihle NT, Williams R, Fenoglio-Preiser $\mathrm{C}$ and Powis G: Stability of phosphoprotein as a biological marker of tumor signaling. Clin Cancer Res 11: 4338, 2005.

12 Espina V, Edmiston KH, Heiby M, Pierobon M, Sciro M, Merritt B, Banks S, Deng J, VanMeter AJ, Geho DH, Pastore L, Sennesh J, Petricoin III EF and Liotta LA: A portrait of tissue phosphoprotein stability in the clinical tissue procurement process. Mol Cell Proteomics 7: 1998-2018, 2008.

13 Mann GB, Fahey VD, Feleppa F and Buchanan MR: Reliance on hormone receptor assays of surgical specimens may compromise outcome in patients with breast cancer. J Clin Oncol 23: 5148-5154, 2008.
Received January 19, 2017

Revised February 24, 2017

Accepted February 28, 2017 